\title{
A study of split-dose cisplatin-based neo-adjuvant chemotherapy in muscle-invasive bladder cancer
}

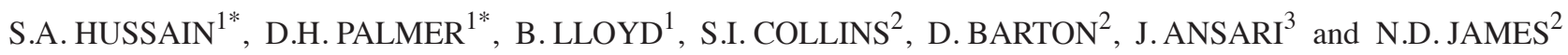 \\ ${ }^{1}$ Department of Molecular and Clinical Cancer Medicine, University of Liverpool, Liverpool; ${ }^{2}$ Cancer Research UK School \\ for Cancer Studies, University of Birmingham, Birmingham; ${ }^{3}$ Beatson West of Scotland Cancer Centre, Glasgow, UK
}

Received October 19, 2011; Accepted January 3, 2012

DOI: $10.3892 / \mathrm{ol} .2012 .563$

\begin{abstract}
The aim of this study was to investigate the outcome of patients with muscle-invasive bladder cancer (MIBC) receiving neo-adjuvant chemotherapy (neo-CT) using a cisplatin-based regimen fractionated on days 1 and 8 of a 21-day cycle prior to organ-preservation (chemoradiation) or cystectomy. Patients with stage T2-T4, N0, M0, transitional cell carcinoma (TCC) of the bladder with a calculated glomerular filtration rate $(\mathrm{GFR}) \geq 40 \mathrm{ml} / \mathrm{min}$ were eligible for inclusion in the study. Neo-CT comprised of gemcitabine $\left(1,000 \mathrm{mg} / \mathrm{m}^{2} \mathrm{~d} 1\right.$, $\mathrm{d} 8, \mathrm{q} 21)$ plus cisplatin $\left(35 \mathrm{mg} / \mathrm{m}^{2} \mathrm{~d} 1, \mathrm{~d} 8, \mathrm{q} 21\right)$ for four cycles. Following the administration of neo-CT, patients underwent surgery or radiotherapy (RT) with or without concurrent chemotherapy (CRT), based on the response to neo-CT and clinician and patient preference. A total of 23 patients were recruited: 21 males and 2 females; median age, 69 years (range, 49-85); stage $\mathrm{T} 2=11, \mathrm{~T} 3 \mathrm{~A}=7, \mathrm{~T} 3 \mathrm{~B}=5$, grade $2=1$, grade $3=22$. One patient progressed prior to neo-CT. In total, 75 cycles of neo-CT were administered. Treatment was well-tolerated with only one episode of neutropenic sepsis. Three of 22 patients developed early progression and did not receive radical treatment. For the remaining 19 patients, choice of definitive treatment (surgery vs. RT/CRT) was based on response to neo-CT. Eight patients had residual disease at cystoscopy following the completion of neo-CT; six patients underwent surgery and two underwent RT/CRT. A total of 11 patients had a complete response (CR) to neo-CT, nine of whom were treated by RT/CRT, with the remaining two declining radical treatment. Median follow-up for alive patients was 57 months (range, 4.4-68.5). Three-year survival was $37 \%$ (95\% CI 17-58\%) and 5-year survival was $31 \%$ (95\% CI $15-52 \%)$. Neo-CT is effective and well-tolerated
\end{abstract}

Correspondence to: Dr Syed A. Hussain, Department of Molecular and Clinical Cancer Medicine, University of Liverpool 5th Floor UCD, Duncan Building, Daulby Street, Liverpool, L69 3GA, UK E-mail: syed.hussain@liverpool.ac.uk

\section{*Contributed equally}

Key words: gemcitabine, neo-adjuvant chemotherapy, split-dose cisplatin outpatient chemotherapy, bladder cancer in MIBC. This split-dose cisplatin regimen facilitates treatment in an outpatient setting and allows inclusion of patients with compromised GFR.

\section{Introduction}

Bladder cancer is the second most common genitourinary malignancy and is a significant cause of morbidity and mortality. Almost $25 \%$ of patients have muscle-invasive disease at presentation $(1,2)$. In North America, the treatment of choice for muscle invasive bladder cancer is radical cystectomy and bilateral pelvic lymph node dissection. Although surgery may be curative, approximately $50 \%$ of all patients with muscle invasive transitional cell carcinoma (TCC) develop metastatic disease within 2 years of cystectomy and subsequently succumb to the disease, presumably due to a significant proportion of patients harboring micrometastatic disease (3). On this basis, neo-adjuvant and adjuvant chemotherapy have been tested in numerous phase II and a few phase III studies (4-7).

There are two principal rationales for neo-adjuvant chemotherapy: i) to improve survival in patients with micrometastatic disease and ii) to preserve the bladder by shrinking the primary tumour to facilitate radiotherapy as an alternative definitive therapy to surgery (8). The results of the South Western Oncology Group (SWOG) neo-adjuvant study revealed an estimated median survival of 6.2 years versus 3.8 years in favour of patients having neo-adjuvant chemotherapy $(\mathrm{P}=0.027)$ (9). Updated results from the UK MRC neo-adjuvant chemotherapy trial show a statistically significant $16 \%$ reduction in the risk of mortality (hazard ratio, $0.84 ; 95 \%$ CI, 0.72-0.99; $\mathrm{P}=0.037$, corresponding to an increase in 10 -year survival from 30 to $36 \%$ ) following neo-adjuvant chemotherapy (10). The SWOG study showed that of the $82 \%$ patients who underwent cystectomy, $38 \%$ had no pathological evidence of disease (9). Patients who achieved pT0 status had a better prognosis than those who did not, although this difference may be accounted for by better disease biology rather than treatment effect. By definition, this study did not address organ-preservation, since the mandated treatment plan was for surgery following neo-adjuvant chemotherapy. The potential disadvantage of neo-adjuvant chemotherapy is correlated to the delay in definitive treatment (cystectomy or chemo-radiotherapy), since this may lead to disease progression in a proportion of cases, with non-responding patients conceivably becoming inoperable. 
Current neo-adjuvant regimens used require in-patient or prolonged hydration schedules, and therefore have a significant impact on patient quality of life and health service resources. Furthermore, these trials have been restricted to patients with well-preserved renal function [typically glomerular filtration rate $(\mathrm{GFR})>60 \mathrm{ml} / \mathrm{min}$ ], thus excluding a significant proportion of bladder cancer patients who are elderly or have ureteric obstruction.

Currently, systemic combination chemotherapy is the only treatment that may result in long-term survival in patients with metastatic disease. The chemosensitivity of bladder cancer is demonstrated by objective response rates of $12-73 \%$ and complete response rates of $0-35 \%$ in patients with metastatic disease (11). Although antitumour activity has been demonstrated with several single agents, the median duration of survival associated with single-agent therapy has generally varied between 4 and 6 months. The median survival time for combination regimens, such as methotrexate plus vinblastine or doxorubicin plus cisplatin, has been 8 months (12). The median survival of patients with metastatic bladder cancer treated with methotrexate, vinblastine, adriamycin and cisplatin (MVAC) chemotherapy or cisplatin, methotrexate and vinblastine (CMV), is approximately 1 year and long-term survival occurs in only a small proportion of patients. Von der Maase et al reported a phase III trial comparing MVAC versus gemcitabine plus cisplatin (GC), both regimens requiring in-patient admission. Patients received (gemcitabine $1,000 \mathrm{mg} / \mathrm{m}^{2}$ on days 1,8 , and 15 ; cisplatin $70 \mathrm{mg} / \mathrm{m}^{2}$ on day 2 ) or standard MVAC every 28 days for a maximum of six cycles. These authors demonstrated that GC provides a similar survival to MVAC with a better safety profile and tolerability (13). Therefore, although this trial was not designed to show equivalence of the two regimens, the results were interpreted as showing therapeutic non-inferiority and GC was adopted as the new standard in view of the better tolerability. With the upper boundary of the confidence interval of the adjusted hazard ratio for survival close to 1.2 , non-inferiority may reasonably be assumed (14). Therefore GC is a valuable alternative for the growing elderly patient population with metastatic bladder cancer, who may derive equal benefit from this regimen as compared to MVAC, but with fewer side effects (15).

Experimental data suggest that a combination of gemcitabine and cisplatin administered using an appropriate schedule (simultaneous or close proximity exposure) are capable of acting synergistically. Synergy may be mediated by the inhibition of ribonucleotide reductase by gemcitabine depleting the deoxynucleotide pool required for DNA replication, thereby inhibiting excision repair of cisplatin-induced DNA crosslinks, or by gemcitabine incorporation into DNA facilitating cisplatin crosslink formation $(16,17)$. Thus, dividing the dose of cisplatin over two weeks may increase the potential for synergistic interaction with gemcitabine. Our previous study conducted in patients with metastatic bladder cancer using GC and exploring a 21-day schedule incorporating fractionated cisplatin showed that dose delays may be avoided by using day 15 as a rest day from chemotherapy and shortening the cycle length compared with widely used 28-day schedules. It also proved that this regimen could be safely delivered in an outpatient setting in a group of patients with GFRs as low as $40 \mathrm{ml} / \mathrm{min}$. The 21-day schedule was found to be less myelosuppressive and thus necessitated fewer treatment delays. Splitting the cisplatin dose over days 1 and 8 also reduced the renal toxicity associated with platinum analogues, extending the potential spectrum of patients treatable with this combination (18). On this basis, the current study was designed to investigate extending the use of this regimen in the neo-adjuvant context.

Patients diagnosed with muscle invasive TCC of the bladder at cystoscopy were eligible to participate in this study.

Patients received up to four cycles of chemotherapy prior to reassessment for definitive radical treatment. Response assessment was undertaken by CT scan, cystoscopy and bladder biopsy, and, then, in consultation with the patient and the multi-disciplinary team, further management included one of the two following options: i) Cystectomy was performed for cases with persistent disease or at clinician or patient request; ii) organ preservation. Patients opting for the organ preservation strategy underwent radical radiotherapy (55 Grays in 20 fractions over 4 weeks) or synchronous chemo-radiotherapy and were eligible for entering into the national randomised phase III trial (BC 2001) that was open and recruiting at the time of this study.

This was a single arm phase II study investigating the toxicity and efficacy of neo-adjuvant chemotherapy using the combination of cisplatin administered on days 1 and 8 of a 21-day schedule with gemcitabine in patients with TCC of the bladder in an outpatient setting.

\section{Patients and methods}

Study objectives. The primary endpoint was response rate. Secondary endpoints were toxicity and survival. The South Birmingham Local Research Ethics Committee approved the study protocol.

Patients. The patients included 21 males and 2 females, with a median age of 69 years (range, 49-85), with histologically confirmed muscle invasive TCC. At least one measurable lesion with a diameter of $\geq 2 \mathrm{~cm}$ was required. Other eligibility criteria were: age $\geq 18$ years; life expectancy $>12$ weeks; WHO performance status $0-2$; adequate haematological function ( $\mathrm{Hb}>10.0 \mathrm{~g} / \mathrm{dl}$; neutrophils $>2.0 \times 10^{9} / 1$; platelets $\left.>100 \times 10^{9} / 1\right)$; adequate renal function (GFR $\geq 40 \mathrm{ml} / \mathrm{min}$ calculated using the Cockcroft formula); adequate liver function [serum bilirubin within normal limits; AST, ALT, ALP $<1.5$ times the upper limit of normal (ULN)]; women of child-bearing potential required a negative pregnancy test prior to entry and both males and females were required to use adequate contraception continuing for 3 months after the study. Patients were given a study information leaflet and provided written, informed consent prior to recruitment into the study.

Study treatment. Pre-hydration with $1000 \mathrm{ml}$ of $0.9 \%$ saline with $20 \mathrm{mmol}$ potassium chloride and $1 \mathrm{~g}$ magnesium sulphate was administered over 2 h. Gemcitabine (1000 mg/ $\mathrm{m}^{2}$ ) was administered in $250 \mathrm{ml}$ of $0.9 \%$ saline and infused over $30 \mathrm{~min}$. This dose was followed by cisplatin $(35 \mathrm{mg} /$ $\mathrm{m}^{2}$ ) administered with hydration using $0.9 \%$ saline $(500 \mathrm{ml})$ over $60 \mathrm{~min}$. All drugs were administered via a peripheral intravenous cannula. Antiemetic therapy comprised $8 \mathrm{mg}$ 
Table I. Dose modification according to haematological toxicity.

\begin{tabular}{lcccc}
\hline & & \multicolumn{2}{c}{ Dose modification } \\
\cline { 4 - 5 } ANC $10^{9} / 1$ & & Platelets $\mathrm{mm}^{3}$ & Gemcitabine & Cisplatin \\
\hline$>1.0$ & and & $\geq 100,000$ & $100 \%$ & $100 \%$ \\
$0.5-1.0$ & or & $50,000-99,000$ & $50 \%$ & $75 \%$ \\
$<0.5$ & or & $<50,000$ & $\begin{array}{c}\text { Delay } \\
\text { Omit }^{\mathrm{b}}\end{array}$ & $\begin{array}{c}\text { Delay } \\
\text { Omit }^{\mathrm{b}}\end{array}$ \\
& & & &
\end{tabular}

aDay 1 treatments were delayed until haematological status allows treatment. This holds for the 100 and $75 \%$ dose. If the delay was $>3$ weeks, the patient was withdrawn from the study. ${ }^{b}$ Day 8 treatment was omitted.

intravenous dexamethasone and $3 \mathrm{mg}$ intravenous granisetron. The total duration of treatment was approximately $4 \mathrm{~h}$. All 23 patients had haematology and clinical chemistry evaluation within $24 \mathrm{~h}$ prior to each treatment, and dose modifications were made as required (Table I). Patients received a maximum of four cycles of treatment depending on response and toxicity.

Statistical considerations. In total, 20 patients were required to allow us to exclude a pathological complete response rate of $<20 \%$. Patients receiving treatment were included in the toxicity assessment. Survival analysis was performed on an intention-to-treat basis. Data were frozen on 8th April, 2011. The response was evaluated using RECIST 1.0 criteria, with a central review of imaging by an independent radiologist. Toxicity data were analysed using simple descriptive statistics. Survival curves were calculated according to the method of Kaplan and Meier (19).

\section{Results}

Patient characteristics. A total of 23 patients entered the trial between September 2004 and July 2007. Patient characteristics are shown in Table II. The median age was 69 years (range, 49-85). Of the 23 patients, 21 were male and 2 female. In total, 11 patients had stage T2 disease and 12 had T3 disease prior to chemotherapy. Ten patients had a calculated GFR of between 40 and $60 \mathrm{ml} / \mathrm{min}$, which may have excluded them from other cisplatin-based protocols.

Toxicity. Toxicity was graded according to Common Toxicity Criteria version 2.0. A total of 75 cycles of chemotherapy were administered. Treatment was well tolerated with only one episode of neutropenic sepsis, which was uncomplicated and was successfully managed with intravenous antibiotics.

Non-haematological toxicity comprised grade 3 nausea and vomiting in 2 patients and grade 3 diarrhoea in 1 patient. Grade 2 renal toxicity was observed in 1 patient with a drop in GFR, although no clinically significant decline in renal function was noted, even in patients with a baseline GFR of $40-60 \mathrm{ml} / \mathrm{min}$. No significant neurotoxicity, ototoxicity or pulmonary toxicity was observed.
Table II. Patient characteristics.

\begin{tabular}{lr} 
Characteristics & Patient no \\
\hline Gender & 21 \\
Male & 2 \\
Female & \\
Age & 69 \\
Median & $(49-85)$ \\
Range & \\
Stage & 11 \\
T2 & 7 \\
T3A & 5 \\
T3B & \\
Grade & \\
2 & 1 \\
3 & 22 \\
Performance status & \\
0 & 0 \\
1 & 14 \\
2 & 9
\end{tabular}

Glomerular filtration rate (GFR)

$40-60 \mathrm{ml} / \mathrm{min}$

$>60 \mathrm{ml} / \mathrm{min}$

Post-chemo treatment

No treatment in view of early progression 4

Radical surgery 6

Radical bladder preservation therapy 11

No further treatment as patient 2

declined further radical treatment

Response. Efficacy analysis was performed on an intention-to-treat basis. One patient progressed with distant metastases prior to initiation of neo-adjuvant chemotherapy and only received palliative care. Three patients developed early progression during neo-adjuvant chemotherapy and did not receive radical treatment. It is plausible that by undergoing neo-adjuvant chemotherapy, these patients were identified as those with biologically aggressive disease and were therefore spared the morbidity of futile radical therapy. For the remaining 19 patients, the choice of definitive treatment (surgery versus organ preservation treatment) was based on response to neo-adjuvant chemotherapy. Eight patients had residual disease on cystoscopy, six of whom underwent cystectomy, while two patients chose RT. A total of 11 patients had a complete pathological response to neo-adjuvant $\mathrm{CT}$, of whom nine underwent radical organ preservation treatment, while two patients declined any further radical treatment and there were no cystectomies in this group.

Survival. At the time of censor, 15 of the 23 patients had succumbed to the disease. The median follow-up for alive patients was 57 months (range, 4.4-68.5). Survival data are shown in Fig. 1. Median survival by intention-to-treat analysis 


\section{Kaplan-Meier survival curve}

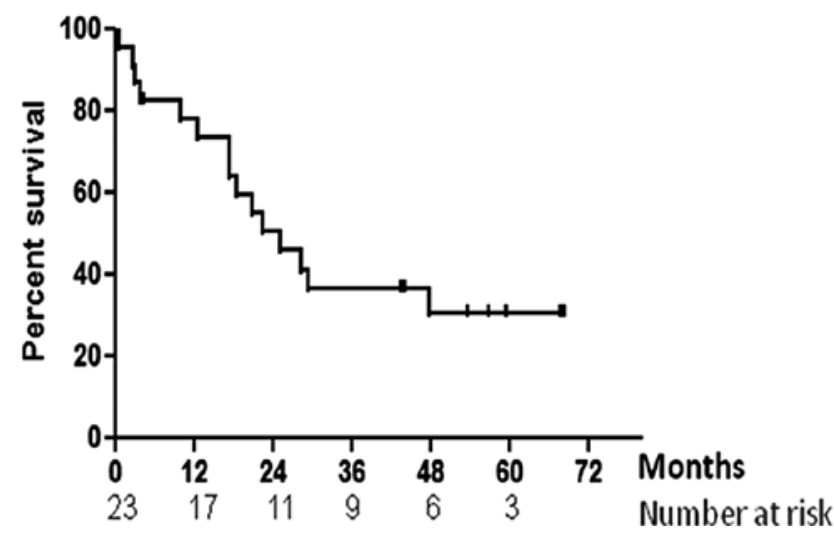

Figure 1. Kaplan-Meier survival data.

was 25.3 months (95\% CI 17.4-47.7). Three-year survival was $37 \%$ (95\% CI 17-58\%) and 5-year survival was 31\% (95\% CI $15-52 \%)$.

\section{Discussion}

Gemcitabine in combination with cisplatin has been widely adopted as standard therapy for advanced muscle invasive bladder cancer in the UK. Adoption of this therapy was based upon better tolerability and non-inferiority compared with older cisplatin-based regimens such as MVAC (13). Cisplatin remains one of the most important drugs in the treatment of advanced TCC of the bladder, although the question of doses administered and scheduling continues to be debated. The required cisplatin dose in a 3 -week cycle is $70-80 \mathrm{mg} / \mathrm{m}^{2}$. This dose requires prolonged intravenous hydration over an 8-h schedule, presenting practical problems for the majority of centres, many of which therefore, administer cisplatin with pre-hydration in an in-patient setting when using doses in excess of $50-60 \mathrm{mg} / \mathrm{m}^{2}$. In the management of advanced bladder cancer, administering chemotherapy in an outpatient setting without compromising efficacy is likely to be beneficial in terms of patient quality of life and health economics. Splitting the cisplatin dose over weeks 1 and 2 and administering concurrently with gemcitabine allows administration over a shorter time in an outpatient setting. Importantly, this maintains the required cisplatin dose intensity. Furthermore, this schedule may facilitate the synergistic interaction between gemcitabine and cisplatin. A number of trials of 28-day regimens report frequent dose modifications and omissions, particularly on day 15 in bladder and lung cancer $(13,20,21)$. Optimising the delivered dose while minimizing dose delays and dose modifications due to toxicity are likely to have an impact on disease-free and overall survival (18).

In this study, we delivered 75 cycles of chemotherapy (150 doses), and encountered only 6 dose reductions (4\%), 3 dose delays by 1 week $(2 \%)$ and 3 dose omissions (2\%) for treatment-related toxicity. The maintenance of dose intensity may have contributed to the encouraging pathological response rates reported here. We included patients with GFR as low as $40 \mathrm{ml} / \mathrm{min}$ with no clinically significant deterioration in renal function. This regimen thus widens the spectrum of patients suitable for cisplatin-based chemotherapy compared with numerous cisplatin-containing trials, which exclude patients with a GFR of less than $60 \mathrm{ml} / \mathrm{min}$. A pathological response rate of $48 \%(11 / 23)$ is encouraging and compares favourably with published data for other neo-adjuvant chemotherapy studies. The SWOG study showed that of the $82 \%$ patients who underwent cystectomy, $38 \%$ had no evidence of disease pathologically (9). Of note is that since patients in the SWOG trial underwent cystectomy, this may have facilitated a more accurate assessment of pathological CR rates compared with the random cystoscopic biopsies performed on our patients undergoing organ-preserving treatment. Median survival of 25.3 months and 5-year survival of $31 \%(95 \%$ CI $15-52 \%$ ) in this group of patients, while limited by the small sample size and wide confidence intervals, is broadly similar to other neo-adjuvant regimens in the published literature. Furthermore, in our study, since the treatment was well tolerated, the definitive treatment was not delayed due to neo-adjuvant chemotherapy related toxicity.

Two large randomised neo-adjuvant studies in muscle invasive bladder cancer have been conducted. Results of both studies showed significant survival advantage $(9,10)$. Although many disciplines in cancer care would consider the data from two completed phase III studies sufficient to alter the standard of care, this does not seem to have occurred for the management of invasive bladder cancer (22). It is the responsibility of urology and oncology colleagues to collaborate in order to provide state-of-the-art care for patients with muscle invasive bladder cancer, which should include neo-adjuvant chemotherapy prior to surgery or organ preservation therapy in fit patients. Personal bias and anecdotal evidence should not overrule evidence from phase III randomised trials. This lack of widespread adoption of neo-adjuvant chemotherapy may relate to the selected patients in clinical trials not reflecting typical muscle invasive bladder cancer patients who may be older and/ or have impaired GFR, which may limit the applicability of some of the chemotherapy regimens. The findings presented in our study suggest that using the approach of split-dose cisplatin is a worthwhile and resource-effective alternative to standard schedules using in-patient bed resources or prolonged hydration, while maintaining dose intensity and good treatment tolerance and efficacy with applicability to a broader range of patients. Further evaluation of this regimen in larger studies in a neo-adjuvant setting is required.

\section{Acknowledgements}

We gratefully acknowledge the support of Lilly UK, for the provision of the study grant.

\section{References}

1. Montie JE, Smith DC and Sandler HM: Carcinoma of the bladder. In: Clinical Oncology. Abbeloff MD (ed). Churchill Livingstone Publications, London, pp1800-1818, 2000.

2. Messing EM, Young TB, Hunt VB, et al: Comparison of bladder cancer outcome in men undergoing hematuria home screening versus those with standard clinical presentation. Urology 45: 387-396, 1995

3. Whitmore WF: Management of invasive bladder neoplasms. Semin Urol 1: 4-10, 1983. 
4. Freiha F, Reese J and Torti FM: A randomised trial of radical cystectomy versus radical cystectomy plus Cisplatin, vinblastine and methotrexate chemotherapy for muscle invasive bladder cancer. J Urol 155: 495-499, 1996.

5. International Collaboration of Trialists: Neoadjuvant cisplatin, methotrexate and vinblastine chemotherapy for muscle invasive bladder cancer: a randomised controlled trial. Lancet 354: 533-540, 1999.

6. Malmstrom PU, Rintala E, Wahlqvist R, Hellstrom P, Hellsten S and Hannisdal E: Five-year follow up of a prospective trial of radical cystectomy and neoadjuvant chemotherapy: Nordic Cystectomy Trial I. The Nordic Cooperative Bladder Cancer Study Group. J Urol 155: 1903-1906, 1996.

7. Stockle M, Wellek S, Meyenburg W, et al: Radical cystectomy with or without adjuvant polychemotherapy for non-organconfined transitional cell carcinoma of the urinary bladder: prognostic impact of lymph node involvement. Urology 48: 868-875, 1996

8. Sternberg CN and Calabrò F: Neo-adjuvant chemotherapy in invasive bladder cancer. World J Urol 19: 94-98, 2001.

9. Grossman, HB, Natale RB, Tangen CM, et al: Neoadjuvant chemotherapy plus cystectomy compared with cystectomy alone for locally advanced bladder cancer. N Engl J Med 349: 859-866, 2003.

10. Griffiths G, Hall R, Sylvester R, Raghavan D and Parmar MK: International phase III trial assessing neoadjuvant cisplatin, methotrexate, and vinblastine chemotherapy for muscle-invasive bladder cancer: long-term results of the BA06 30894 trial. J Clin Oncol 29: 2171-2177, 2011.

11. Juffs HG, Moore MJ and Tannock IF: The role of systemic chemotherapy in the management of muscle - invasive bladder cancer. Lancet Oncol 3: 738-747, 2002.

12. Sternberg C, Marini L and Calabrò F: Systemic chemotherapy of bladder cancer. In: Bladder Cancer: Biology and Management. Skinner DG and Syrigos KN (eds). Oxford University Press, New York, pp299-315, 1999.
13. Von der Maase H, Hansen SW, Roberts JT, et al: Gemcitabine and cisplatin versus methotrexate, vinblastine, doxorubicin, and cisplatin in advanced or metastatic bladder cancer: results of a large, randomised, multinational, multicenter, phase III study. J Clin Oncol 18: 3068-3077, 2000.

14. de Wit R, Bochner BH and Scher HI: Treatment for metastatic urothelial cancer. In: American Society of Clinical Oncology educational book. Perry CM (ed). pp226-244, 2001.

15. Hussain SA and James ND: The role of systemic chemotherapy in advanced and metastatic bladder cancer. Lancet Oncol 4 489-497, 2003.

16. Bergman AM, Ruiz van Haperen VW, Veerman G, Kuiper CM and Peters GJ: Synergistic interaction between cisplatin and gemcitabine in vitro. Clin Cancer Res. 2: 521-530, 1996.

17. Crul M, van Waardenburg RC, Bocxe S, van Eijndhoven MA, Pluim D, Beijnen JH and Schellens JH: DNA repair mechanisms involved in gemcitabine cytotoxicity and in the interaction between gemcitabine and cisplatin. Biochem Pharmacol 65: 275-282, 2003.

18. Hussain SA, Stocken DD, Riley P, et al: A phase I/II study of gemcitabine and fractionated cisplatin in an outpatient setting using a 21-day schedule in patients with advanced and metastatic bladder cancer. Br J Cancer 91: 844-849, 2004

19. Kaplan EL and Meier P: Non-parametric estimation from incomplete observations. J Am Statist Association 53: 457-458, 1958.

20. Lippe P, Tummarello D, Monterubbianesi MC, et al: Weekly gemcitabine and cisplatin in advanced non small cell lung cancer: A phase II study. Ann Oncol 10: 217-221, 1999.

21. Berardi R, Porfiri E, Scartozzi M, et al: Elderly patients with advanced non small cell lung cancer: A phase II study with weekly cisplatin and gemcitabine. Oncology 65: 198-203, 2003.

22. Bochner BH: Chemotherapy: standardizing the care of invasive bladder cancer. Nat Rev Clin Oncol 8: 454-455, 2011. 